\title{
Association of hematocrit with blood pressure and hypertension
}

\author{
Marzieh Emamian $^{1, \dagger}$ | Seyed Mahdi Hasanian ${ }^{2,3, \dagger}$ | Maryam Tayefi,4,5, \\ Moniba Bijari $^{4}$ | Faeze Movahedian far ${ }^{4}$ | Mojtaba Shafiee ${ }^{4}$ | Amir Avan ${ }^{1, \dagger}$ \\ Alireza Heidari-Bakavoli6,† | Mohsen Moohebati ${ }^{6}$ | Mahmoud Ebrahimi ${ }^{6}$ | \\ Sousan Darroudi $^{1}$ | Parvin Zamani ${ }^{7}$ | Mahmoud Reza Azarpazhooh ${ }^{6}$ | Mohsen Nematy ${ }^{1}$ | \\ Mohammad Safarian $^{1}$ | Gordon A. Ferns ${ }^{8}$ | Habibollah Esmaeili ${ }^{5}$ | \\ Mohammad Reza Parizadeh $^{1,2}$ | Majid Ghayour-Mobarhan ${ }^{1,2,6}$ \\ ${ }^{1}$ Metabolic Syndrome Research Center, School of Medicine, Mashhad University of Medical Sciences, Mashhad, Iran \\ ${ }^{2}$ Department of Medical Biochemistry, School of Medicine, Mashhad University of Medical Sciences, Mashhad, Iran \\ ${ }^{3}$ Microanatomy Research Center, Mashhad University of Medical Sciences, Mashhad, Iran \\ ${ }^{4}$ Student Research Committee, School of Medicine, Mashhad University of Medical Sciences, Mashhad, Iran \\ ${ }^{5}$ Department of Biostatistics \& Epidemiology, School of Health, Management \& Social Determinants of Health Research Center, Mashhad University of Medical \\ Sciences, Mashhad, Iran \\ ${ }^{6}$ Cardiovascular Research Center, School of Medicine, Mashhad University of Medical Sciences, Mashhad, Iran \\ ${ }^{7}$ Department of Medical Biotechnology, Faculty of Medicine, Mashhad University of Medical Sciences, Mashhad, Iran \\ ${ }^{8}$ Brighton \& Sussex Medical School, Division of Medical Education, Falmer, Brighton, Sussex, UK
}

\section{Correspondence}

Majid Ghayour-Mobarhan, Metabolic Syndrome Research Center, School of Medicine, Mashhad University of Medical Sciences, Mashhad, Iran.

Email: ghayourm@mums.ac.ir

Mohammad Reza Parizadeh, Department of Medical Biochemistry, School of Medicine, Mashhad University of Medical Sciences, Mashhad, Iran.

Email: ParizadehMR@mums.ac.ir

Funding information

This study was support by grant from

Mashhad University of Medical Sciences.
Background: Hypertension (HTN) is a risk factor for stroke, renal failure, and cardiovascular disease. The association between biochemical and hematological parameters with high blood pressure may provide a more precise approach to risk prediction conferred by HTN in these patients.

Objective: The aim of current study was to explore whether biochemical and hematological parameters are associated with HTN in a cohort study with a 7-year follow-up. Materials and Methods: A total of 9808 individuals were enrolled and recruited as part of the Mashhad Stroke and Heart Atherosclerotic Disorders (MASHAD) cohort study, and biochemical and hematological factors were measured in all subjects. Univariate and multivariate logistic regression analysis were performed to determine the association of biochemical and hematological parameters with HTN.

Results: Several biochemical parameters including fasting plasma glucose (FBG), serum high-sensitivity C-reactive protein (hs-CRP), triglyceride (TG), total cholesterol (TC), low-density lipoprotein cholesterol (LDL-C), and uric acid were increased in hypertensive participants. In contrast, serum high-density lipoprotein cholesterol (HDL-C) was lower in hypertensive individuals. Furthermore, we demonstrated that hematological parameters including white blood cell (WBC) count, red blood cell (RBC) count, hemoglobin (HGB), hematocrit (HCT), and mean corpuscular hemoglobin $(\mathrm{MCH})$ were higher in the hypertensive group compared to the control group. But mean corpuscular 
volume (MCV), and red cell distribution width (RDW), were decreased in the hypertensive group. Furthermore, our results strongly suggested that among these parameters, hematocrit was the independent risk factor for hypertension in the population.

Conclusion: We demonstrated the association of altered biochemical and hematological factors with hypertension supporting the value of emerging markers for early prediction of high blood pressure in prone individuals.

\section{KEYWORDS}

biochemical risk factors, cardiovascular diseases, hematological parameters, hypertension

\section{1 | INTRODUCTION}

Hypertension is defined as a systolic pressure $\geq 140 \mathrm{~mm} \mathrm{Hg}$ or a diastolic pressure $\geq 90 \mathrm{~mm} \mathrm{Hg}^{1}$ The prevalence of hypertension is different among various geographic regions ${ }^{2}$ and is increasing worldwide. Thus, identification of novel biomarkers could help in early detection and effective prevention of hypertension complications in high risk patients.

The relationship between high blood pressure and biochemical risk factors has been studied in detail. Most studies in this field have focused on the association of lipid profile with hypertension and their effect on the severity of clinical presentations in cardiovascular diseases. ${ }^{3-5}$ But little is known about the association of hematological parameters with HTN. Recently it has been demonstrated that elevated white blood cell (WBC) count, especially neutrophils, is associated with hypertension. ${ }^{6-8}$ Moreover, high levels of red cell distribution width (RDW), variability in the size of circulating erythrocytes, is associated with hypertension. ${ }^{9}$ Consistent with these findings, hematocrit (HCT), a major determinant of blood viscosity, is associated with the risk of high blood pressure and is considered as a risk factor for HTN. ${ }^{10-12}$ Furthermore, the association of hemoglobin (HGB) level in the pathogenesis of hypertension related diseases has been investigated. ${ }^{13,14}$

While some research has been carried out on the relationship between biochemical and hematological factors with hypertension, in most of these studies only one or two factors were studied in a small population, thus role of these biochemical and hematological parameters as independent risk factors for predicting hypertension and severity of clinical presentations is controversial. In the present study we explored the association of almost all of biochemical and hematological parameters with risk of HTN occurrence in 9808 individuals in a cohort study to provide a new insight regarding the relationship between biochemical and hematological markers with risk of HTN.

\section{2 | MATERIALS AND METHODS}

\section{1 | Population}

In current study, 9808, 3934 men and 5874 women, were recruited as part of the Mashhad Stroke and Heart Atherosclerotic Disorders
(MASHAD) Study using a cluster-randomized-assigned during 20072008 , as described previously. ${ }^{15}$ Recruits had no known history of infectious diseases, nor a family history of stroke, myocardial infarction, and diabetes mellitus. They were divided into two groups according to the report of American Heart Association (23.7\% in the hypertensive and $76.3 \%$ in the control group). we considered the patients with a systolic pressure $\geq 140 \mathrm{~mm} \mathrm{Hg}$ or a diastolic pressure $\geq 90 \mathrm{~mm} \mathrm{Hg}$ as hypertensive Informed consent was obtained from all participants using protocols approved by the Ethics Committee of the MUMS. ${ }^{15}$

\section{2 | Measurement of blood pressure}

We measured SBP and DBP by sphygmomanometer twice in exactly the same manner. It was measured on the left arm when the individuals remained seated at rest for 15 minutes. We took the third measurement and averaged the two closest readings, if the first two readings differ by more than $15 \mathrm{~mm} \mathrm{Hg}$ in diastolic or more than $25 \mathrm{~mm} \mathrm{Hg}$ in systolic blood pressure.

\section{3 | Anthropometric and biochemical measurements}

Anthropometric parameters, including height, body weight, Body mass index (BMI) and waist circumference (WC) were evaluated in all the subjected as previously described. ${ }^{16,17}$ Biochemical parameters, including total cholesterol (TC), high-density lipoprotein cholesterol (HDL-C), low-density lipoprotein cholesterol (LDL-C) and triglyceride (TG), C-reactive protein (CRP), fasting blood glucose (FBG) and uric acid were determined as described previously. ${ }^{18,19}$

\section{4 | Measurements of hematological markers}

Hematological factors, including white blood cell (WBC), red blood cell (RBC), hemoglobin (HGB), hematocrit (HCT), mean corpuscular volume (MCV), mean corpuscular hemoglobin $(\mathrm{MCH})$, mean corpuscular hemoglobin concentration (MCHC), red cell distribution width (RDW), platelet count (PLT), platelet distribution width (PDW) were measured in all the subjects, as described previously. ${ }^{20}$ 


\section{5 | Statistical analysis}

Data analysis was carried out using SPSS-18 software (SPSS Inc., IL, USA). The normality of data was evaluated using Kolmogorov-Smirnov test. Descriptive statistics including mean, frequency, and standard deviation (SD) were determined for all variables and expressed as Mean \pm standard deviation (SD) for variables with normally distribution or median \pm IQR for not normally distributed variables. For normally distributed variables, Student's $t$ test was applied to compare clinical and baseline demographics characteristics between groups. The Mann-Whitney $U$ test was used for continuous non-normal variables. For categorical parameters, Chi-square or Fisher exact tests were used. Logistic regression analysis was used to evaluate the association of hematological parameters with HTN. All the analyses were twosided and $P$ value $<.05$ was considered as significant.

\section{3 | RESULTS}

\subsection{General characteristics of the subjects}

Clinical and demographic characteristics of participants are presented in Table 1. Almost one-fourth of the participants ( 23.7\%) had HTN. The mean age was $52.06 \pm 7.92$ and $46.85 \pm 7.97$ years for HTN and control groups respectively. Our results demonstrated that the levels of BMI, WC, SBP, DBP, FBG, hs-CRP, and Uric acid were significantly increased in HTN group, compared to the normotensive (NTN) one. But no statistically differences were found on sex and height between HTN and NTN groups (Table 1). Moreover, compared to the NTN group TC, TG and LDL-C were markedly increased whereas HDL-C decreased in the HTN individuals (Table 1).

\subsection{Comparison of hematological parameters between groups}

We also compared the level of different hematological parameters of the participants to compare these results between HTN and NTN groups. Results presented in Table 2 clearly demonstrated that compared with the control group, hypertensive individuals had significantly higher level of WBC, RBC, HGB, HCT $(P<.001)$ and $\mathrm{MCH}$ $(P<.05)$. In contrast, level of MCV and RDW were lower in the HTN group $(P<.05)$ (Table 2$)$. But there were no significant differences between the groups with regard to mean corpuscular hemoglobin concentration (MCHC), PDW or PLT.

\section{3 | Demographic, biochemical, and hematological indices as risk factors for hypertension}

Logistic regression analysis was performed to determine the odds ratio (OR) of the association between demographic, biochemical and hematological parameters with HTN (Table 3). Results obtained from univariate analysis for biochemical risk factors demonstrated that BMI, FBG, TC, LDL, Uric Acid, hs-CRP are associated with HTN. To further investigate the association of hematological parameters
TABLE 1 Comparison of demographic and biochemical characteristics between hypertensive (HTN) and normotensive (NTN) individuals

\begin{tabular}{|lllc}
\hline Parameters & HTN & NTN & P-value \\
\hline Age, year & $52.06 \pm 7.92$ & $46.85 \pm 7.97$ & $<.001$ \\
\hline Gender, N (\%) & Male: $(41.4 \%)$ & Male: (39.7\%) & .14 \\
\hline & Female: $(58.6 \%)$ & Female: (60.3\%) & \\
\hline BMI $\left(\mathrm{kg} / \mathrm{m}^{2}\right)$ & $29.28 \pm 4.67$ & $27.47 \pm 4.67$ & $<.001$ \\
\hline SBP $(\mathrm{mm} \mathrm{Hg})$ & $145.6 \pm 19.13$ & $114.47 \pm 11.64$ & $<.001$ \\
\hline DBP $(\mathrm{mm} \mathrm{Hg})$ & $93.11 \pm 11.1$ & $74.81 \pm 8$ & $<.001$ \\
\hline WC $(\mathrm{cm})$ & $99.33 \pm 11.57$ & $93.94 \pm 11.91$ & $<.001$ \\
\hline Weight $(\mathrm{kg})$ & $75.18 \pm 13.06$ & $70.73 \pm 12.65$ & $<.001$ \\
\hline Height $(\mathrm{m})$ & $1.60 \pm 0.094$ & $1.61 \pm .091$ & .26 \\
\hline TC $(\mathrm{mg} / \mathrm{dL})$ & $199.84 \pm 55.65$ & $189.71 \pm 61.28$ & $<.001$ \\
\hline LDL-C $(\mathrm{mg} / \mathrm{dL})$ & $120.22 \pm 36.95$ & $115.41 \pm 34.66$ & $<.001$ \\
\hline hs-CRP $(\mathrm{mg} / \mathrm{dL})$ & $1.98(3.19)$ & $1.55(2.34)$ & $<.001$ \\
\hline HDL-C $(\mathrm{mg} / \mathrm{dL})$ & $43 \pm 10.03$ & $42.82 \pm 9.92$ & .45 \\
\hline FBG $(\mathrm{mg} / \mathrm{dL})$ & $100.97 \pm 45.95$ & $90.11 \pm 36.67$ & $<.001$ \\
\hline TG $(\mathrm{mg} / \mathrm{dL})$ & $137(93)$ & $115(85)$ & $<.001$ \\
\hline Uric acid & $4.93 \pm 1.39$ & $4.58 \pm 1.4$ & $<.001$ \\
\hline
\end{tabular}

BMI, Body mass index; SBP, systolic blood pressure; DBP, diastolic blood pressure; WC, waist circumference; TC, total cholesterol; LDL-C, lowdensity lipoprotein cholesterol; hs-CRP, high-sensitivity $C$ reactive protein; HDL-C, high-density lipoprotein cholesterol; FBG, fasting blood glucose; TG, triglyceride.

Values are presented as the mean $\pm \mathrm{SD}$ or median and IQR for normally or non-normally distributed variables, respectively. Student's $t$ test and Mann-Whitney $U$ test were used to compare differences between two groups.

TABLE 2 Comparison of the serum level of hematological parameters between hypertensive (HTN) and those with normal blood pressure (NTN)

\begin{tabular}{|llll|}
\hline Parameters & HTN & NTN & P-value \\
\hline WBC $\left(10^{9} / \mathrm{L}\right)$ & $6.21 \pm 1.55$ & $6.05 \pm 1.57$ & $<.001$ \\
\hline RBC $\left(10^{12} / \mathrm{L}\right)$ & $4.93 \pm 0.48$ & $4.83 \pm 0.49$ & $<.001$ \\
\hline $\mathrm{HGB}(\mathrm{g} / \mathrm{dL})$ & $13.89 \pm 1.54$ & $13.68 \pm 1.82$ & $<.001$ \\
\hline $\mathrm{HCT}(\%)$ & $41.72 \pm 4.08$ & $41.08 \pm 5.26$ & $<.001$ \\
\hline $\mathrm{MCV}(\mathrm{fl})$ & $84.58 \pm 6$ & $85.01 \pm 6$ & .003 \\
\hline $\mathrm{MCH}(\mathrm{pg} / \mathrm{cell})$ & $28.25 \pm 2.39$ & $28.36 \pm 2.74$ & $<.05$ \\
\hline $\mathrm{RDW}(\%)$ & $13.82 \pm 1.01$ & $13.91 \pm 1.05$ & .002 \\
\hline $\mathrm{MCHC}(\mathrm{g} / \mathrm{dL})$ & $33.24 \pm 1.78$ & $33.25 \pm 1.64$ & .77 \\
\hline $\mathrm{PLT}\left(10^{9} / \mathrm{L}\right)$ & $231.46 \pm 63.7$ & $229.46 \pm 60.25$ & .17 \\
\hline $\mathrm{PDW}(\%)$ & $12.89 \pm 3.39$ & $12.74 \pm 1.99$ & .06 \\
\hline
\end{tabular}

WBC, White blood cell; RBC, red blood cell; HGB, hemoglobin; HCT, hematocrit; $\mathrm{MCV}$, mean corpuscular volume; $\mathrm{MCH}$, mean corpuscular hemoglobin; RDW, red cell distribution width; $\mathrm{MCHC}$, mean corpuscular hemoglobin concentration; PLT, platelet count; PDW, platelet distribution width.

Values are presented as the mean \pm SD. Student's $t$ test was used to compare differences between two groups. 


\begin{tabular}{|lllll|} 
& Univariate OR & $P$ value & Multivariate OR & $P$ value \\
\hline Age & $1.08(1.07-1.09)$ & $<.001$ & $0.99(0.98-1.006)$ & .28 \\
\hline High BMI & $2.13(1.89-2.39)$ & $<.001$ & $0.86(0.965-1.14)$ & .29 \\
\hline Uric acid & $1.88(1.15-1.23)$ & $<.001$ & $0.99(0.91-1.07)$ & .85 \\
\hline hs-CRP & $1.01(1.005-1.02)$ & $<.001$ & $1.00(0.98-1.02)$ & .98 \\
\hline High FBG & $2.04(1.82-2.27)$ & $<.001$ & $1.23(0.93-1.63)$ & .14 \\
\hline High TC & $0.6(0.55-0.66)$ & $<.001$ & $0.97(0.75-1.23)$ & .78 \\
\hline High LDL-C & $1.27(1.15-1.40)$ & $<.001$ & $0.81(0.64-1.02)$ & .07 \\
\hline WBC & $1.07(1.04-1.10)$ & $<.001$ & $1.01(0.94-1.09)$ & .82 \\
\hline RBC & $1.51(1.37-1.66)$ & $<.001$ & $0.92(0.661-1.28)$ & .61 \\
\hline HGB & $1.08(1.04-1.11)$ & $<.001$ & $1.00(0.95-1.05)$ & .97 \\
\hline HCT & $1.03(1.02-1.04)$ & $<.001$ & $1.02(1.003-1.04)$ & .02 \\
\hline MCV & $0.99(0.98-1.00)$ & .003 & $0.98(0.96-1.002)$ & .08 \\
\hline RDW & $0.98(0.96-0.99)$ & .001 & $0.98(0.94-1.02)$ & .29 \\
\hline
\end{tabular}

TABLE 3 Univariate and multivariate logistic regression analysis was performed to determine the odds ratio (OR) of the association between demographic, biochemical and hematological parameters with HTN

Body mass index (BMI), high-sensitivity $\mathrm{C}$ reactive protein (hs-CRP), fasting blood glucose (FBG), total cholesterol (TC), low-density lipoprotein cholesterol (LDL-C), White blood cell (WBC), red blood cell (RBC), hemoglobin (HGB), hematocrit (HCT), mean corpuscular volume (MCV), red cell distribution width (RDW).

Multivariate analysis was adjusted with the variables were significantly different between two groups.

with hypertension, logistic regression analyses were performed (Table 3). Our results strongly suggested the association of HCT with HTN as an independent predictive risk factor for hypertension (Table 3).

\section{4 | DISCUSSION}

We have investigated the association of biochemical and hematological factors with hypertension in a large Iranian population. We have found that level of TC, LDL-C, TG, FBG, hs-CRP and Uric acid were significantly increased in HTN individuals compared to the normotensive group. We also measured the association between hematological parameters with HTN and our results clearly demonstrated that hypertensive individuals had higher level of WBC, RBC, $\mathrm{HGB}, \mathrm{MCH}$, and $\mathrm{HCT}$ compared to the control one. In contrast, level of MCV and RDW were lower in the HTN group $(P<.05)$. To determine the odds ratio for these parameters logistic regression analysis was performed and we found that hematocrit (HCT) is an independent risk factor for hypertension which could be a novel prognostic marker for early detection of hypertension in high risk patients.

In support of our findings, Jae et al. reported an association between HCT and incidence of hypertension during a mean follow-up of 5 years. However, their study population was only restricted to men. ${ }^{10}$ Moreover, in another study Liu et al. in a large cross-sectional study found an independent association between HCT and prehypertension in Chinese population. ${ }^{11}$ Tripolino et al. also found that the subjects with prehypertension had significantly higher hematocrit, blood and plasma viscosity levels than those of the normotensive subjects, whereas they were comparable to those of the hypertensive subjects. ${ }^{21}$ Also it is suggested that the prevalence of hypertension was at least two times greater for individuals with Hct levels higher by
10 units. $^{22}$ Nakanishi et al., in a cohort of Japanese men, found that hematocrit was closely associated with risk for hypertension, after controls for other potential factors. ${ }^{23}$ Another study reported that mean arterial blood pressure (MAP) was related to Hct in postmenopausal women. ${ }^{24}$ Another study showed that Hct was significantly associated with increased Homeostasis model assessment of insulin resistance (HOMA-IR), in Japanese community dwelling persons. The authors also observed a dose-dependent relationship between Hct levels and the components of metabolic syndrome. ${ }^{25}$ In support of the importance of Hct, Zeng et al. also demonstrated that higher Hct was independently associated with the incidence of hyperuricemia (HU), even within the normal range. ${ }^{26}$ Increase in HCT is usually accompanied by high blood viscosity and increase in RBC count. It has been shown that erythrocyte count is increased in hypertensive subjects which would increase blood pressure through imposing additional load on cardiovascular system. ${ }^{27,28}$ Consistent with these findings, HGB, major cytosolic protein in erythrocytes, was markedly increased in HTN group compared to the NTN population. HGB through limiting the nitric oxide availability for vascular smooth muscle cells induced vasoconstriction which explains the positive association between elevated level of HGB with HTN. ${ }^{29}$ The association between HGB and HTN was also investigated in a recent cross-sectional study on Korean population in where they showed a positive association between HGB level with SBP and DBP. ${ }^{14}$ Some studies showed that mean corpuscular volume (MCV) is a hypertension-related risk factor, ${ }^{28,30,31}$ whereas others showed no relationship between MCV and hypertension. ${ }^{32}$ Babu et al. in a case-control study containing 100 hypertensive patients and 100 normotensive subjects demonstrated that MCV appears to be inversely related to systolic and diastolic blood pressure. ${ }^{28}$ Consistent with these findings, we showed that compared to the control group MCV is decreased in HTN individuals. Decrease in $\mathrm{MCV}$ is an adaptive mechanism to decrease RBC-induced 
hypertension and viscosity without compromising blood flow. ${ }^{28,31,33}$ In our study, there was no significant difference in mean corpuscular hemoglobin concentration (MCHC) between HTN and NTN groups. These results are in contrast to those studies reported an increased or decreased level of MCHC in HTN subjects. ${ }^{28,32}$ The reason of this inconsistency may be related to the differences in population size and ethnicity.

There is growing body of evidence suggesting the association of WBC count with hypertension. ${ }^{8,34}$ For instance, Nakashiki et al. in a cohort study on 3776 Japanese male office workers aged 23-49 years, demonstrated that WBC count is a risk factor for hypertension. ${ }^{34}$ Moreover, WBC count is an independent predictor of cardiovascular morbidity in hypertensive patients. ${ }^{35}$ In another cohort study, Shankar et al. found that increased WBC count is associated with hypertension in white skin population. ${ }^{8}$ Our findings are in agreement with these studies suggesting that WBC count is elevated in hypertensive individuals and is associated with high blood pressure.

There are also evidence suggesting that RDW is a potential predictor of mortality and morbidity in cardiovascular diseases. ${ }^{36,37}$ Elevated RDW has been associated with adverse clinical outcomes in patients with hypertension, ${ }^{38}$ coronary heart disease ${ }^{39}$ and pulmonary hypertension. ${ }^{40}$ In a prospective study by chavon et al. RDW was an important prognostic biomarker for pulmonary hypertension complications. ${ }^{41}$ Increased level of RDW may act as a marker of systemic inflammation and oxidative stress which are important biological mechanisms in both initiation and progression of hypertension. ${ }^{9}$ Systemic inflammation can cause bone marrow dysfunction leading to release of immature erythrocytes and subsequent anisocytosis which increase the level of RDW, as well. ${ }^{42}$ Conversely, in our study level of RDW was higher among normotensive individuals. This can be explained, at least in part, by considering the role of gene polymorphisms and gene deletions in regulating hypertension in different populations. ${ }^{43-47}$ Further studies could investigate the association of candidate gene polymorphisms on hypertension in Iranian population.

A major strength of the present study is that it was performed in a large population which provides a new insight regarding the relationship between biochemical and hematological parameters with risk of HTN in a representative sample of Iran which have a high prevalence of hypertension and CVD. Our findings suggested that prevention and treatment of HTN should give a priority for decreasing the prevalence of CVD and its associated diseases. However, the main limitation was cross-sectional study design and possible influence of ethnicity and diet pattern of our population, supporting further prospective studies to explore the impact of emerging markers of hematological and biochemical factors on the risk of developing HTN and cardiovascular diseases.

In conclusion, in the light of worldwide epidemic of hypertension and its effect on cardiovascular mortality/morbidity, early detection and effective prevention of hypertension is one of the current public health challenges. As we show the association between hypertension and biochemical and hematological factors, routine blood test for high risk individuals, may be an effective prevention strategy.

\section{REFERENCES}

1. Smith SC, Blair SN, Bonow RO, et al. AHA/ACC guidelines for preventing heart attack and death in patients with atherosclerotic cardiovascular disease: 2001 update: a statement for healthcare professionals from the American Heart Association and the American College of Cardiology. J Am Coll Cardiol. 2001;38:1581-1583.

2. Kearney PM, Whelton M, Reynolds K, Muntner P, Whelton PK, He J. Global burden of hypertension: analysis of worldwide data. Lancet 2005;365:217-223.

3. Sander GE, Giles TD. Hypertension and lipids: lipid factors in the hypertension syndrome. Curr Hypertens Rep. 2002;4:458-463.

4. Halperin RO, Sesso HD, Ma J, Buring JE, Stampfer MJ, Gaziano JM. Dyslipidemia and the risk of incident hypertension in men. Hypertension. 2006;47:45-50.

5. Kostis JB. The importance of managing hypertension and dyslipidemia to decrease cardiovascular disease. Cardiovasc Drugs Ther. 2007;21:297-309.

6. Kawada T, Morihashi M, Ueda H, Sirato T. Neutrophil cell count is related to hypertension in workers: a cross-sectional study. Vasc Dis Prev. 2007;4:225-228.

7. Orakzai R, Orakzai S, Nasir K, et al. Association of white blood cell count with systolic blood pressure within the normotensive range. J Hum Hypertens. 2006;20:341-347.

8. Shankar A, Klein BE, Klein R. Relationship between white blood cell count and incident hypertension. Am J Hypertens. 2004;17:233-239.

9. Fici F, Celik T, Balta S, et al. Comparative effects of nebivolol and metoprolol on red cell distribution width and neutrophil/lymphocyte ratio in patients with newly diagnosed essential hypertension. $J$ Cardiovasc Pharmacol. 2013;62:388-393.

10. Jae SY, Kurl S, Laukkanen JA, et al. Higher blood hematocrit predicts hypertension in men. J Hypertens. 2014;32:245-250.

11. Liu X, Liang J, Qiu Q, et al. Association of hematocrit and prehypertension among Chinese adults: the CRC study. Cell Biochem Biophys. 2015;71:1123-1128.

12. Paul L, Jeemon P, Hewitt J, et al. Hematocrit predicts long-term mortality in a nonlinear and sex-specific manner in hypertensive adults. Hypertension. 2012;60:631-638.

13. Atsma F, Veldhuizen I, de Kort W, van Kraaij M, Pasker-de Jong P, Deinum J. Hemoglobin level is positively associated with blood pressure in a large cohort of healthy individuals. Hypertension 2012;60:936-941.

14. Lee SG, Rim JH, Kim JH. Association of hemoglobin levels with blood pressure and hypertension in a large population-based study: the Korea National Health and Nutrition Examination Surveys 20082011. Clin Chim Acta. 2015;438:12-18.

15. Ghayour-Mobarhan M, Moohebati M, Esmaily H, et al. Mashhad stroke and heart atherosclerotic disorder (MASHAD) study: design, baseline characteristics and 10-year cardiovascular risk estimation. Int J Public Health. 2015;60:561-572.

16. Zomorrodian D, Khajavi-Rad A, Avan A, et al. Metabolic syndrome components as markers to prognosticate the risk of developing chronic kidney disease: evidence-based study with 6492 individuals. J Epidemiol Community Health 2015;69:594-598.

17. Emamian M, Avan A, Pasdar A, et al. The lipoprotein lipase S447X and cholesteryl ester transfer protein rs5882 polymorphisms and their relationship with lipid profile in human serum of obese individuals. Gene. 2015;558:195-199.

18. Mirhafez SR, Zarifian A, Ebrahimi M, et al. Relationship between serum cytokine and growth factor concentrations and coronary artery disease. Clin Biochem. 2015;48:575-580.

19. Mirhafez SR, Pasdar A, Avan A, et al. Cytokine and growth factor profiling in patients with the metabolic syndrome. Br J Nutr. 2015;113:1911-1919. 
20. Mardan-Nika M, Pasdar A, Jamialahmadi K, et al. Association of heat shock protein70-2 (HSP70-2) gene polymorphism with obesity. Ann Hum Biol 2016;43:542-546.

21. Tripolino C, Gnasso A, Carallo C, Scavelli FB, Irace C. Hemorheological profiles of subjects with prehypertension. Hypertens Res 2016;39:519-523.

22. Cirillo M, Laurenzi M, Trevisan M, Stamler J. Hematocrit, blood pressure, and hypertension. The Gubbio Population Study. Hypertension. 1992;20:319-326.

23. Nakanishi N, Yoshida H, Okamoto M, et al. Hematocrit and risk for hypertension in middle-aged Japanese male office workers. Ind Health. 2001;39:17-20.

24. Vázquez BS, Vázquez MS, Intaglietta M, De Faire U, Fagrell B, Cabrales P. Hematocrit and mean arterial blood pressure in pre-and postmenopause women. Vasc Health Risk Manag. 2009;5:483-488.

25. Kawamoto R, Tabara Y, Kohara K, et al. Hematological parameters are associated with metabolic syndrome in Japanese community-dwelling persons. Endocrine. 2013;43:334-341.

26. Zeng C, Wei J, Yang T, et al. Higher blood hematocrit predicts hyperuricemia: a prospective study of 62897 person-years of follow-up. Sci Rep 2015;5:13765.

27. Wu S, Lin H, Zhang C, et al. Association between erythrocyte parameters and metabolic syndrome in urban Han Chinese: a longitudinal cohort study. BMC public health. 2013;13:989.

28. Babu KR, Solepure A, Shaikh R. Comparison of hematological parameters in primary hypertensives and normotensives of Sangareddy. Int J Biomed Res. 2015;6:309-315

29. Cabrales P, Han G, Nacharaju P, Friedman AJ, Friedman JM. Reversa of hemoglobin-induced vasoconstriction with sustained release of nitric oxide. Am J Physiol Heart Circ Physiol. 2011;300:H49-H56.

30. Haltmayer M, Mueller T, Luft C, Poelz W, Haidinger D. Erythrocyte mean corpuscular volume associated with severity of peripheral arterial disease: an angiographic evaluation. Ann Vasc Surg. 2002;16:474-479.

31. Karabulut A, Karadag A. Clinical implication of hematological indices in the essential hypertension. World J Hypertens. 2015;5:93-97.

32. Persson SU, Gustavsson CG, Larsson H, Persson S. Studies on blood rheology in patients with primary pulmonary hypertension. Angiology. 1991;42:836-842.

33. Sharp DS, Curb JD, Schatz IJ, et al. Mean red cell volume as a correlate of blood pressure. Circulation. 1996;93:1677-1684.

34. Nakanishi N, Sato M, Shirai K, Suzuki K, Tatara K. White blood cell count as a risk factor for hypertension; a study of Japanese male office workers. J Hypertens. 2002;20:851-857.

35. Brown DW, Giles WH, Croft JB. White blood cell count: an independent predictor of coronary heart disease mortality among a national cohort. J Clin Epidemiol. 2001;54:316-322.
36. Cavusoglu E, Chopra V, Gupta A, et al. Relation between red blood cell distribution width (RDW) and all-cause mortality at two years in an unselected population referred for coronary angiography. Int J Cardiol. 2010;141:141-146.

37. Perlstein TS, Weuve J, Pfeffer MA, Beckman JA. Red blood cell distribution width and mortality risk in a community-based prospective cohort. Arch Intern Med. 2009;169:588-594.

38. Özcan F, Turak O, Durak A, et al. Red cell distribution width and inflammation in patients with non-dipper hypertension. Blood Press. 2013;22:80-85.

39. Tonelli M, Sacks F, Arnold M, Moye L, Davis B, Pfeffer M. Relation between red blood cell distribution width and cardiovascular event rate in people with coronary disease. Circulation. 2008;117:163-168.

40. Abul Y, Ozsu S, Korkmaz A, Bulbul Y, Orem A, Ozlu T. Red cell distribution width: a new predictor for chronic thromboembolic pulmonary hypertension after pulmonary embolism. Chron Respir Dis. 2014;11:73-81.

41. Hampole CV, Mehrotra AK, Thenappan T, Gomberg-Maitland M, Shah SJ. Usefulness of red cell distribution width as a prognostic marker in pulmonary hypertension. Am J Cardiol. 2009;104:868-872.

42. Fornal M, Wizner B, Cwynar M, et al. Association of red blood cell distribution width, inflammation markers and morphological as well as rheological erythrocyte parameters with target organ damage in hypertension. Clin Hemorheol Micro. 2014;56:325-335.

43. Lobmeyer MT, Wang L, Zineh I, et al. Polymorphisms in genes coding for GRK2 and GRK5 and response differences in antihypertensive treated patients. Pharmacogenet Genomics. 2011;21:42.

44. Patel SK, Velkoska E, Freeman M, Wai B, Lancefield TF, Burrell LM From gene to protein-experimental and clinical studies of ACE2 in blood pressure control and arterial hypertension. Front Physiol 2014;5:227.

45. Rudnicki M, Mayer G. Significance of genetic polymorphisms of the renin-angiotensin-aldosterone system in cardiovascular and renal disease. Pharmacogenomics. 2009;10:463-476.

46. Santulli G, Cipolletta E, Sorriento D, et al. CaMK4 gene deletion induces hypertension. J Am Heart Assoc. 2012;1:e001081.

47. Santulli G, Trimarco B, laccarino G. G-protein-coupled receptor kinase 2 and hypertension: molecular insights and pathophysiological mechanisms. High Blood Press Cardiovasc Prev. 2013;20:5-12.

How to cite this article: Emamian M, Hasanian SM, Tayefi M, et al. Association of hematocrit levels with blood pressure and hypertension. J Clin Lab Anal. 2017;31:e22124. https://doi. org/10.1002/jcla.22124 